\title{
RELATING GROUP TOPOLOGIES BY THEIR CONTINUOUS POINTS
}

\author{
KEVIN J. SHARPE
}

\begin{abstract}
ABSTRACr. Let $x$ be a point in a topological group $G$, and for each integer $n$, let $(1 / n) x$ be the set $\{y: n y=x\}$ in $G$. Then I call $x$ a continuous point if for positive integers $n$, the subsets $(1 / n) x$ are nonvoid and eventually intersect each neighbourhood of the identity 0 . I prove the following result and from it three corollaries. Let $G$ be a divisible abelian group such that $(1 / n) 0=\{0\}$ for some integer $n \geqslant 2$. Suppose there are two group topologies $\mathbb{Q}_{1}$ and $\mathbb{Q}_{2}$ defined on $G$ and that $G$ is $Q_{2}$-locally compact and $\sigma$-compact, and define $\omega_{2}$ to be the outer measure derived from the Haar measure $\mu_{2}$ on $\left(G, \mathbb{Q}_{2}\right)$. Also suppose that the ratio of the $\mathbb{Q}_{2}$-measure of $\{n x: x \in A\}$ to the $\mathbb{Q}_{2}$-measure of $A$, for any $\mathbb{Q}_{2}$-Borel-measurable set $A$ (the ratio is the same for any such $A$ with finite measure), does not exceed 1 . Then for each $\mathbb{Q}_{2}$-Borel-measurable set $A$ with nonvoid $\mathbb{Q}_{1}$-interior, $\mu_{2}(A)$ $>\omega_{2}\left(W_{1}\right), W_{1}$ being the subgroup of all points in $G$ which are $\mathbb{Q}_{1^{-}}$ continuous.
\end{abstract}

The study of compact group topologies for the real line gave rise to the rather interesting questions posed by D. N Hawley [1] and answered by me for $R^{N}$ [4]. I propose to present now a generalization of the proofs in [4], something which supplies the basis for the study of what I call the continuous points in a topological group (see [5]). This work forms part of a Ph.D. thesis submitted to La Trobe University in Melbourne, Australia, and was done under the supervision of Dr. Graham Elton.

Definitions. Let $G$ be a group (I write my groups additively) and $A$ a subset of $G$. It is possible to define two kinds of " $n$ th-multiples" of the set $A$ :

$$
n A=\left\{x_{1}+x_{2}+\cdots+x_{n}: x_{1}, x_{2}, \ldots, x_{n} \in A\right\},
$$

for $n$ a positive integer, and

$$
{ }^{\circ} n A=\{n x: x \in A\},
$$

for $n$ any integer.

An element $x$ of $G$ is divisible (in $G$ ) if for each positive integer $n$ there is a $y$ in $G$ satisfying $x=n y$. If every element of $G$ is divisible in $G$, then $G$ is said to be divisible. To avoid excess of writing, I put $(1 / n) x=\{y: n y=x\}$, and for $A$ a subset of $G,{ }^{\circ}(1 / n) A=\{y: n y \in A\}$.

Now consider $G$ to be a topological group. I call a divisible element $x$ of $G$

Received by the editors September 13,1973 and, in revised form, April 12, 1974 and February 11, 1975.

AMS (MOS) subject classifications (1970). Primary 22B99; Secondary 28A05.

Key words and phrases. Continuous points, topological group, Haar measure, Hawley property, uniquely rooted group. 
a continuous point if the subsets $(1 / n) x$, for positive integers $n$, eventually intersect each neighbourhood of the identity. In other words, if $A$ is a neighbourhood of the identity and $x$ is a continuous point, there is a positive integer $N$ such that for all $n>N,(1 / n) x \cap A \neq \varnothing$. I designate the collection of continuous points in $G$ by $W$, and if $G$ is abelian, $W$ is a subgroup.

Most of this work concerns groups which are divisible and abelian; these I call $d a$ groups for short. I am also concerned with the torsion-free property in that it involves this idea: $G$ is uniquely nth-rooted if $y_{1}$ and $y_{2}$ in $G$ are such that $n y_{1}=n y_{2}$, then $y_{1}=y_{2}(n$ is a positive integer). If $G$ is an abelian group, then the uniquely $n$ th-rooted property is equivalent to $G$ 's containing no points, except the identity 0 , whose $n$ th-multiple is 0 . (Note that if $x$ in $G$ is uniquely $n$ th-rooted, $(1 / n) x$ contains at most one point, and I take $(1 / n) x$ to be that point.)

First I want to show that ${ }^{\circ} n A$ and ${ }^{\circ}(1 / n) A$ are Borel (-measurable) for a Borel set $A$ and a positive integer $n$.

LEMMA 1. If $G$ is a da $\sigma$-compact locally compact group, then, for each positive integer $n$, the function $f_{n}: x \rightarrow n x$, for all $x$ in $G$, is an open and continuous homomorphism of $G$ onto $G$.

Proof. That $f_{n}$ is continuous follows simply from the definition of a topological group (see [3, p. 96, part A]), and the open property follows from (5.29) in [2, p. 42].

It is true then that, in any topological group, not only translates and inverses of Borel sets are again Borel, but also that the $n$ th-multiples of Borel sets are Borel when the group is $\sigma$-compact, locally compact and uniquely $n$ th-rooted.

To my main train of thought. I want to build up to the fact that for certain groups $G$ with Haar measure $\mu, \mu\left({ }^{\circ} n A\right) \leqslant \mu(A)$ for a positive integer $n$ and for all $A$ in $\Re$, the $\sigma$-algebra of all Borel sets. To do this define $\mu^{n}$ by $\mu^{n}(A)=\mu\left({ }^{\circ} n A\right)$ for all $A$ in $\Re$. If $G$ is da, uniquely $n$ th-rooted, locally compact and $\sigma$-compact, then $\mu^{n}$ is a Haar measure on $G$; for instance

$$
\mu^{n}(x+A)=\mu\left({ }^{\circ} n(x+A)\right)=\mu\left(n x+{ }^{\circ} n A\right)=\mu\left({ }^{\circ} n A\right)=\mu^{n}(A),
$$

for all $x$ in $G$ and $A$ in $\Re$. But the Haar measure on $G$ is essentially unique, and so there is a positive real $c_{n}$ such that $\mu^{n}=c_{n} \mu$. It can be shown that $c_{n}$ is the product of integer powers of the prime factors of $n$, but more important for this study, it can be shown that $c_{n}$ is not dependent on the particular Haar measure chosen for the topology. If $c_{n} \leqslant 1$, then $\mu\left({ }^{\circ} n A\right)=\mu^{n}(A) \leqslant \mu(A)$ for all $A$ in $\mathfrak{R}$, and this would be the case if, for instance, $G$ contains a compact open subgroup. To summarize these results:

LemMa 2. Let $G$ be a da, uniquely nth-rooted, $\sigma$-compact, locally compact group with $c_{n} \leqslant 1$ for some integer $n \geqslant 2$. Then $\mu\left({ }^{\circ} n A\right) \leqslant \mu(A)$ for all Borel sets $A$ and for a Haar measure $\mu$.

Now to my main result.

THEOREM 3. Let $G$ be a da, uniquely nth-rooted group for some integer $n \geqslant 2$. Suppose there are two group topologies $\mathbb{Q}_{1}$ and $\mathbb{Q}_{2}$ defined on $G$, such that $(G$, 
$\mathbb{Q}_{2}$ ) is locally compact, $\sigma$-compact and for it $c_{n} \leqslant 1$, and define $\omega_{2}$ to be the outer measure derived from the Haar measure $\mu_{2}$ on $\left(G, \mathbb{Q}_{2}\right)$. Then for any $\mathbb{Q}_{2}$-Borel set $A$ with $Q_{1}$-interior containing 0 ,

$$
\omega_{2}\left(W_{1}\right) \leqslant \mu_{2}\left(\bigcup_{v=0}^{\infty}{ }^{\circ} n^{v}\left(\bigcap_{m=0}^{\infty}{ }^{\circ} n^{m} A\right)\right)=\mu_{2}\left(\bigcap_{m=0}^{\infty}{ }^{\circ} n^{m} A\right) \leqslant \mu_{2}(A),
$$

where $W_{1}$ is the subgroup of all the points in $G$ which are $\mathbb{Q}_{1}$-continuous.

Proof. Let $A$ be such an $\mathbb{Q}_{2}$-Borel set which contains the $\mathbb{Q}_{1}$-open neighbourhood $B$ of 0 . Then let $K=\cap_{m=0}^{\infty} n^{m} A$, and $L=\cup_{v=0}^{\infty}{ }^{\circ} n^{v} K$; from Lemma 1 it follows that $K$ and, hence, $L$ are $\mathbb{Q}_{2}$-Borel.

As each $x$ in $W_{1}$ is an $\mathbb{Q}_{1}$-continuous point, the sets $(1 / u) x$, for positive integers $u$, eventually intersect $B$ and, hence, they eventually intersect $A$. That is: there is a positive integer $U$ such that for any $u>U,(1 / u) x \cap A \neq \varnothing$. Take $q$ an integer such that $n^{q}>U$. Then for all $m \geqslant 0, n^{m} n^{q}>U$ and $\left(1 / n^{m} n^{q}\right) x \cap A \neq \varnothing$. Now for any positive integers $a$ and $c$,

$$
\begin{aligned}
(1 / a)[(1 / c) x] & ={ }^{\circ}(1 / a)\{y \in G: c y=x\} \\
= & \{z \in G: a z=y \text { and } c y=x \text { for some } y \in G\} \\
= & \{z \in G: a c z=x\}=(1 / a c) x .
\end{aligned}
$$

Hence $\left(1 / n^{m}\right)\left[\left(1 / n^{q}\right) x\right] \cap A \neq \varnothing$ for all $m \geqslant 0$. This and the fact that $G$ is uniquely $n^{q}$ th-rooted put $\left(1 / n^{q}\right) x$ in ${ }^{\circ} n^{m} A$, for all $m \geqslant 0$, and thus in $K$. So $x$ is in ${ }^{\circ} n^{q} K \subseteq L$, and $W_{1} \subseteq L$.

I want to show that $\left\{{ }^{\circ} n^{v} K\right\}_{v=0}^{\infty}$ is an ordered chain of subsets, that is, ${ }^{\circ} n^{v} K \subseteq{ }^{\circ} n^{v+1} K$ for any positive integer $v$. If $x$ is in ${ }^{\circ} n^{v} K$, then $\left(1 / n^{v}\right) x$ is in $K$ and $\left(1 / n^{v}\right) x$ is in ${ }^{\circ} n^{m} A$ for all $m \geqslant 0$. Hence, $\left(1 / n^{v}\right) x$ is an element of ${ }^{\circ} n\left({ }^{\circ} n^{m} A\right)={ }^{\circ} n^{m+1} A$ for all $m \geqslant 0$, and taking $n$ th-roots, $(1 / n)\left[\left(1 / n^{0}\right) x\right]$ is an element of ${ }^{\circ} n^{m} A$ for all $m \geqslant 0$. So $\left(1 / n^{v+1}\right) x$ is in $\cap_{m=0}^{\infty}{ }^{\circ} n^{m} A=K$ and $x$ in ${ }^{\circ} n^{v+1} K$, making ${ }^{\circ} n^{0} K \subseteq{ }^{\circ} n^{v+1} K$.

The fact that ${ }^{\circ} n^{v} K \subseteq{ }^{\circ} n^{v+1} K$ for all $v \geqslant 0$ means that

$$
\mu_{2}\left(\bigcup_{v=0}^{\infty}{ }^{\circ} n^{v} K\right)=\lim _{v \rightarrow \infty} \mu_{2}\left({ }^{\circ} n^{v} K\right)
$$

which is less than $\mu_{2}(K)$ by Lemma 2 . Combining this with the facts that $W_{1} \subseteq L, K \subseteq L$, and $A \supseteq K$, gives $\omega_{2}\left(W_{1}\right) \leqslant \mu_{2}(L)=\mu_{2}(K) \leqslant \mu_{2}(A)$, the required result.

There are three corollaries from this result, the second two of which form the basis of further work in this field (see [5]). The definition stated below arises from a generalization of the condition Hawley was interested in for the reals, and is used in Corollary 6.

Corollary 4. Let $G, \mathbb{Q}_{1}$ and $\mathbb{Q}_{2}$ be as in Theorem 3 , except that for the second topology $c_{n}<1$. Then $W_{1}$ is $\mathbb{Q}_{2}$-negligible if there is an $\mathbb{Q}_{2}$-Borel set with finite $\mathbb{Q}_{2}$-measure and having a nonvoid $\mathbb{Q}_{1}$-interior.

COROLlaRY 5. Let $G$ be a da, uniquely nth-rooted, nondiscrete topological group for some integer $n \geqslant 2$, and which is locally compact, $\sigma$-compact and has $c_{n} \leqslant 1$. Then the subgroup of continuous points in $G$ is negligible. 
Proof. Putting $Q_{1}=Q_{2}$ in Theorem 3 implies that every open set has measure at least the outer measure of the subgroup of continuous points. But by regularity, since a point has zero measure, there are sets open in $G$ with arbitrarily small measures.

Definition. Suppose there are two topologies $Q_{1}$ and $Q_{2}$ defined on some space $X$. Then $Q_{2}$ is Hawley with respect to $Q_{1}$ if, given any $Q_{2}$-Borel set, either it or its complement is dense in $\left(X, Q_{1}\right)$.

COROllaRY 6. Let $G$ be a da, uniquely nth-rooted group for some integer $n \geqslant 2$. Suppose there are two group topologies $Q_{1}$ and $Q_{2}$ defined on $G$, and $\mathbb{Q}_{2}$ causes $G$ to be compact. Then $\mathbb{Q}_{2}$ is Hawley with respect to $\mathbb{Q}_{1}$ if the subgroup of $\mathbb{Q}_{1}$-continuous points is not $\mathbb{Q}_{2}$-negligible.

Proof. In any compact and connected group, a nonnegligible subgroup has outer measure 1 . Now by applying Theorem 3 with $c_{n}=1$ to our present group, it can be seen that every $\mathbb{Q}_{2}$-Borel set with nonvoid $\mathbb{Q}_{1}$-interior must have $\mathbb{Q}_{2}$-measure 1 . Thus the $\mathbb{Q}_{2}$-measure of $G$ is two times what it should be if an $Q_{2}$-Borel set and its complement are both not dense in $\left(G, Q_{1}\right)$.

If $Q_{2}$ is Hawley with respect to $\mathbb{Q}_{1}$ for two topologies $\mathbb{Q}_{1}$ and $\mathbb{Q}_{2}$ on some space $X$, then the only functions from $X$ to a Hausdorff space both $Q_{1^{-}}$ continuous and $Q_{2}$-Borel-measurable are the constant functions. This can be proved in exactly the same way as Theorem 4 in [4]. However, it is not possible to remove the " $Q_{1}$-continuous" and make it " $Q_{1}$-Borel-measurable", for if $\left(X, Q_{1}\right)$ and $\left(X, Q_{2}\right)$ are Hausdorff spaces and $X$ contains two distinct points $x$ and $y$, the map $f: X \rightarrow\{x, y\}$ defined by $f(x)=x$ and $f(z)=y$ if $z$ is in $\{x\}^{\prime}$, is $\mathbb{Q}_{1}$ - and $\mathbb{Q}_{2}$-Borel-measurable, but is not a constant function.

\section{REFERENCES}

1. D. N. Hawley, Compact group topologies for R, Proc. Amer. Math. Soc. 30 (1971), 566-572. MR 43 \#7548.

2. E. Hewitt and K. A. Ross, Abstract harmonic analysis. Vol. I: Structure of topological groups. Integration theory, group representations, Die Grundlehren der math. Wissenschaften, Band 115, Academic Press, New York; Springer-Verlag, Berlin, 1963. MR 28 \# 158.

3. L. S. Pontrjagin, Topological groups, 2nd ed., GITTL, Moscow, 1954; English transl., Gordon and Breach, New York, 1966. MR 17, 171; MR 34 \# 1439.

4. K. J. Sharpe, Two properties of $R^{N}$ with a compact group topology, Proc. Amer. Math. Soc. 34 (1972), 267-269. MR 45 \# 2083.

5 . _ Continuous points in topological groups (submitted).

Department of Mathematics, La Trobe University, Melbourne, Australia

Current address: Episcopal Divinity School, Cambridge, Massachusetts 02138 